\title{
The impact of optic nerve movement on optic nerve magnetic resonance diffusion parameters
}

\begin{abstract}
Authors:
Anand Moodley ${ }^{1}$

William I.D. Rae ${ }^{2}$

Yarish Brijmohan ${ }^{3}$

Miranda Durand ${ }^{4}$

Catherine Connolly ${ }^{5}$

Andrew Michowicz ${ }^{6}$

Ahmed Bhigjee

\section{Affiliations:}

${ }^{1}$ Department of Neurology,

Grey's Hospital, University of

KwaZulu-Natal, South Africa

${ }^{2}$ Department of Medical

Physics, University of the

Free State, South Africa

${ }^{3}$ Department of Electrical, Electronic and Computer Engineering, University of KwaZulu-Natal, South Africa

${ }^{4}$ Department of Radiology, Grey's Hospital, South Africa

${ }^{5}$ Biostatistics Unit,

Medical Research Council,

South Africa

${ }^{6}$ Department of Medicine,

Edendale Hospital,

South Africa

${ }^{7}$ Department of Neurology, University of KwaZulu-Natal, South Africa
\end{abstract}

Correspondence to:

Anand Moodley

Email:

anand.moodley1@gmail.com

Postal address:

PO Box 13833

Cascades 3202, South Africa

Dates:

Received: 05 Mar. 2014

Accepted: 30 July 2014

Published: 15 Dec. 2014

How to cite this article: Moodley, A, Rae, WID,

Brijmohan $Y$, et al. The

impact of optic nerve

movement on optic nerve

magnetic resonance diffusion

parameters. S Afr J Rad.

2014;18(1); Art. \#596, 6 pages.

http://dx.doi.org/10.4102/

sajr.v18i1.596

\section{Read online:}

Background: Optic nerve diffusion imaging is a useful investigational tool of optic nerve microstructure, but is limited by eye-movement-induced optic nerve movement and artifacts from surrounding cerebrospinal fluid, fat, bone and air. Attempts at improving patient cooperation, thus voluntarily limiting eye movement during a standard diffusion imaging sequence, are usually futile. The aim of this study was to establish the impact of optic nerve movement on clinical diffusion parameters of the optic nerve.

Method: Twenty-nine healthy volunteers with intact vision and intact conjugate gaze were recruited and subjected to magnetic resonance diffusion-weighted imaging (DWI) and diffusion-tensor imaging (DTI) of the optic nerves. Twenty right eyes had nerve tracking done using single-shot echo-planar imaging at 20 time points over 3 minutes. Optic nerve movement measurements were correlated with diffusion parameters of apparent diffusion coefficient (ADC), mean diffusivity (MD), fractional anisotropy (FA) and anisotropic index (AI) using Spearman's rank correlation.

Results: No significant correlations were noted between optic nerve movement parameters and ADC in the axial plane and MD of the optic nerve. Low to moderate negative correlations were noted between optic nerve movement parameters and AI and FA and positive correlation with ADC in the radial plane.

Conclusion: Optic nerve movement documented during the timespan of standard diffusion sequences (DWI and DTI) has a negative effect on the anisotropic diffusion parameters of the optic nerve. With greater eye movement, optic nerve diffusion appears less anisotropic owing to greater radial diffusion.

\section{Introduction}

Optic nerve $(\mathrm{ON})$ diffusion imaging has gained prominence as a useful investigational tool for optic nerve microstructure and pathology. ${ }^{1,2,3}$ Whilst initially developed to demonstrate the effects of chronic optic neuritis on ON structure, its use in recent studies has revealed early changes in acute optic neuritis, too. ${ }^{4}$ The anticipated benefit of detecting these early changes is better understanding of the pathophysiology of processes affecting the optic nerve and possibly the development of better strategies to prevent and reverse such damage. ON diffusion imaging has also been used both qualitatively and quantitatively for ischaemic optic neuropathy, glaucoma and Cryptococcusinduced visual loss. ${ }^{5,6,7}$ Different pulse sequences and imaging protocols for $\mathrm{ON}$ diffusion imaging have been used (Table 1). The lack of standardisation of imaging parameters has had apparently little effect on the quantitative assessment of $\mathrm{ON}$ diffusion parameters. Good consistency for such parameters has been determined using the different techniques of Iwasawa's intravoxel incoherent motion (IVIM), ${ }^{8}$ Wheeler-Kingshott's zonal oblique multislice echo planar imaging (ZOOM EPI), ${ }^{9}$ Chabert's non-Carr-Purcell-Meiboom-Gill fast spin echo (non-CPMG FSE) ${ }^{10}$ and our coronal oblique method. ${ }^{7}$ Each method, unfortunately, has its own limitation. The ON is surrounded firstly by cerebrospinal fluid (CSF) within the optic nerve sheath, and then by fat within a bony orbit which is bordered by paranasal sinuses containing air. In most of the ON diffusion techniques, fluid-attenuated inversion recovery (FLAIR) and fat saturation are utilised to minimise the CSF and fat artifact surrounding the nerve, respectively. We used fat saturation (spectral presaturation with inversion recovery [SPIR]) to suppress the fat signal and a high, but clinically widely used, diffusion gradient $\left(b=1000 \mathrm{~s} / \mathrm{mm}^{2}\right)$ instead of FLAIR, to reduce the CSF artifact. ${ }^{7}$ The benefit of this method was improved identification of the $\mathrm{ON}$ on $b=0 \mathrm{~s} / \mathrm{mm}^{2}$ images. On coronal oblique imaging, the nerve appears centrally hypo-intense and is surrounded by hyper-intense CSF in the peri-optic space. Susceptibility artifact from the bone and air are minimised by using sensitivity encoding (SENSE), averaging and Rayleigh noise reduction. ${ }^{9}$ ON motion, however, cannot be entirely eradicated, but merely decreased by asking the subject to be calm, listen to relaxing music, and focus on a target placed in his central field of view. We asked subjects to focus on an orange

Copyright: @ 2014. The Authors. Licensee: AOSIS OpenJournals. This work is licensed under the Creative Commons Attribution License. 
TABLE 1: Summary of demographic data (where available), diffusion sequences and parameters of the optic nerve.

\begin{tabular}{|c|c|c|c|c|c|c|c|c|}
\hline $\begin{array}{l}\text { Summary of demographic data } \\
\text { (where available), diffusion } \\
\text { sequences and paramaters of } \\
\text { the optic nerve diffusion }\end{array}$ & $\begin{array}{l}\text { The present } \\
\text { study }\end{array}$ & $\begin{array}{l}\text { Wheeler- } \\
\text { Kingshott DWI } \\
\text { study }\end{array}$ & $\begin{array}{l}\text { Iwasawa DWI } \\
\text { study*,8 }\end{array}$ & $\begin{array}{l}\text { Wheeler- } \\
\text { Kingshott DTI } \\
\text { study }^{14}\end{array}$ & $\begin{array}{l}\text { Trip DTI } \\
\text { study }^{2}\end{array}$ & $\begin{array}{l}\text { Chabert DTI } \\
\text { study }^{10}\end{array}$ & $\begin{array}{l}\text { Xu DTI } \\
\text { study }\end{array}$ & $\begin{array}{l}\text { Kolbe DTI } \\
\text { study }^{12}\end{array}$ \\
\hline Volunteers recruited & 29 & 3 & 7 & 10 & 15 & 9 & 12 & 20 \\
\hline Number of eyes analysed & 52 & - & - & - & - & - & - & - \\
\hline Diffusion Sequence & $\begin{array}{l}\text { Coronal } \\
\text { oblique }\end{array}$ & ZOOM-EPI & IVIM & ZOOM-EPI & ZOOM-EPI & $\begin{array}{l}\text { Non-CPMG } \\
\text { FSE }\end{array}$ & IVI-EPI & $\begin{array}{l}\text { Coronal } \\
\text { oblique }\end{array}$ \\
\hline FAT suppression & Yes & Yes & No & Yes & Yes & No & Yes & Yes \\
\hline CSF suppression & No & Yes & No & Yes & Yes & No & No & Yes \\
\hline Mean age (range) & $30(21-46)$ & - & $30.9(28-34)$ & - & - & - & - & - \\
\hline Number of female subjects (\%) & $14(48 \%)$ & - & & - & - & - & - & - \\
\hline $\begin{array}{l}\text { ADC optic nerve: } \\
\text { mean }(\mathrm{SD}) \times 10^{-3} \mathrm{~mm}^{2} / \mathrm{s}\end{array}$ & $1.30 \pm(0.4)$ & $1.058 \pm(0.1)$ & $\begin{array}{l}0.98 \pm(0.74) Y \\
1.56 \pm(0.68) Z\end{array}$ & - & - & - & - & - \\
\hline $\begin{array}{l}\text { MD optic nerve: } \\
\text { mean (SD) } \times 10^{-3} \mathrm{~mm}^{2} / \mathrm{s}\end{array}$ & $1.35 \pm(0.5)$ & - & - & $1.22 \pm(0.2)$ & $1.08 \pm(0.17)$ & $1.1 \pm(0.2)$ & $1.09 \pm(0.2)$ & $1.25 \pm(0.14)$ \\
\hline FA optic nerve: mean (SD) & $0.44 \pm(0.1)$ & - & - & $0.61 \pm(0.1)$ & $0.67 \pm(0.09)$ & $0.49 \pm(0.06)$ & $0.46 \pm(0.15)$ & $0.38 \pm(0.05)$ \\
\hline
\end{tabular}

SD, standard deviation; $A D C$, apparent diffusion coefficient measured using diffusion-weighted imaging; $M D$, mean diffusivity measured using diffusion tensor imaging; $F A$, fractional anisotropy; $\mathrm{Al}$, anisotropic index calculated as $2 \lambda_{1} /\left(\lambda_{2}+\lambda_{3}\right)$, where $\lambda_{1}=$ axial diffusion and $\lambda_{2}, \lambda_{3}=$ radial diffusion; IQR, interquartile range; ZOOM EPI, zonal oblique multi-slice echo-planar imaging; IVIM, intravoxel incoherent motion; Non-CPMG FSE, non-Carr-Purcell-Meiboom-Gill fast spin echo; IVI-EPI, inner volume imaging - echo-planar imaging.

*,Iwasawa et al. obtained $A D C$ in the $Y$ and $\mathrm{Z}$ axes.

sticker placed overhead on the MRI machine. Nevertheless, eye movement (voluntary and/or involuntary) and therefore ON movement continues, and it is not uncommon with singleshot EPI to image an apparent 'double nerve' as a result of movement. The effect of this movement on $\mathrm{ON}$ diffusion parameters has not been previously investigated.

To investigate the pathophysiology of various ON disorders, including those which derange the CSF flow dynamics of the nerve and surrounding CSF, it would be useful to have some quantitative measure of the flow within the CSF and $\mathrm{ON}$, and therefore it is necessary to look at factors, such as movement, that influence the accuracy of quantitation. All research done so far on $\mathrm{ON}$ diffusion has not factored in the influence of $\mathrm{ON}$ movement on the diffusion parameters. Diffusion in the radial plane is more likely to be affected by ON movement than diffusion in the axial direction, since displacement of the nerve occurs predominantly in the radial plane. Consequently, the ratio of axial to radial diffusion (i.e. the anisotropic index [AI]) will be affected by the degree of movement. Anxious and encephalopathic patients are more likely to have more eye movement and scanning movements than lucid patients, which may affect the AI and perhaps other diffusion parameters.

The first aim of this study was to determine the amount of movement of the $\mathrm{ON}$ that occurs during the timespan of a standard diffusion imaging study when attempts were made to reduce movement. The second aim was to determine the effect of this movement on the diffusion parameters, namely apparent diffusion coefficient (ADC), mean diffusivity (MD), fractional anisotropy (FA) and AI of the ON.

\section{Method}

Full ethical approval for the study was obtained from the Grey's Hospital Ethics Committee and the University of
KwaZulu-Natal Ethics Department. Informed consent was obtained from healthy volunteers with intact visual acuity and ocular motor function demonstrating preserved conjugate eye movements in the horizontal and vertical axes. Each volunteer underwent full neuro-ophthalmological examination, to ensure normality of visual function, and an MRI scan of the orbits and brain. A 1.5T Philips Gyroscan was used for magnetic resonance (MR) diffusion-weighted imaging (DWI) and diffusion-tensor imaging (DTI) of the brain and ONs using the coronal oblique technique for each nerve as previously reported. ${ }^{7}$ A clinically relevant diffusion gradient of $b=1000 \mathrm{~s} / \mathrm{mm}^{2}$ was selected for the orthogonal three-axis DWI acquisitions, and fat-saturated (SPIR) singleshot EPI using SENSE without cardiac gating was used. EPI was acquired using echo time $(\mathrm{TE})=93 \mathrm{~ms}$, repetition time $(\mathrm{TR})=3000 \mathrm{~ms}, 180 \mathrm{~mm}$ field of view $(\mathrm{FOV})$, matrix = $112 \times 128$ and $4 \mathrm{~mm}$ slice thickness. DTI was obtained in 15 directions and longitudinal, axial and mean diffusivity values were selected for analysis. Twenty-nine volunteers were recruited who demonstrated bilateral visual acuity of $6 / 6$ or better, full range of extra-ocular movement and preserved conjugate eye movements. ON tracking using single-shot EPI followed immediately after DWI and DTI of the ON. An MRI-compatible eye-tracking device was not available for this study. We therefore opted for MR EPI optic nerve tracking, which is limited in that the nerve tracking could not be performed at the same time as diffusion acquisition. However, subjects were made to lie on the table for at least 25 minutes prior to the acquisition of DWI and DTI of the ONs, when standard brain imaging of FLAIR, T1W, T2W and DWI were acquired. This period prior to diffusion imaging of the ONs and EPI tracking of the ONs allowed the subjects to settle down and acclimatise to the MRI milieu. We therefore postulated that when ON diffusion and EPI tracking were acquired, the subjects' emotional state and level of anxiety were generally stable and equivalent, hence resulting in a similar amount of eye movement during both acquisitions. Conjugate eye movements were essential 
as only the tracking of the right $\mathrm{ON}$ was done. ON movement measurements so obtained could therefore be correlated to diffusion parameters from the right and left eyes. Twenty snapshots of the ON were done in 3 minutes, which was the equivalent duration of the standard $\mathrm{ON}$ diffusion study. Images were spaced at 9-second intervals from each other. During both studies, the subjects, while relaxing and listening to light music, was asked to fixate on an orange sticker placed in their field of view on the inner anterior surface of the MRI scanner. ONs selected for analysis were those that were easily identified on the $b=0 \mathrm{~s} / \mathrm{mm}^{2}$ coronal oblique diffusion images.

ON tracking was done on the image series acquired for 20/29 volunteers using the 20 images obtained for each subject over the 3-minute period. The 20 images were acquired in a plane perpendicular to the long axis of the nerve, and positioned to include the orbital segment of the nerve midway between the optic canal and the globe where minimal artifact from surrounding bone and paranasal sinus air was present. A target of 1 pixel size $(1.6 \mathrm{~mm} \times 1.6 \mathrm{~mm})$ was placed in the centre of the $\mathrm{ON}$ in the coronal oblique image (Figure 1) for all 20 images. The position of the $\mathrm{ON}$ in image 1 was labelled as the reference point $\left(\mathrm{x}_{1} \mathrm{y}_{1}\right)$ with which all subsequent 19 images were compared. The ON movement parameters (viz. total distance moved from the reference point, total area of displacement and displacement in the $x$ and $y$ axes) were recorded. Figures $2 a-2 c$ show displacement of the $\mathrm{ON}$ in the $\mathrm{x}$ axis, $\mathrm{y}$ axis and the $\mathrm{x}-\mathrm{y}$ plane. All simulations were performed in MATLAB R2009b. The total distance (sum of all the 19 distances recorded on the images from the starting point) from the reference point was calculated using the formula:

distance $\left(\mathrm{x}_{20}, \mathrm{y}_{20} ; \mathrm{x}_{19}, \mathrm{y}_{19}\right)+$ distance $\left(\mathrm{x}_{19}, \mathrm{y}_{19} ; \mathrm{x}_{18}, \mathrm{y}_{18}\right)+\ldots$

distance $\left(\mathrm{x}_{2}, \mathrm{y}_{2}, \mathrm{x}_{1}, \mathrm{y}_{1}\right)$,

where distance $(\mathrm{a}, \mathrm{b} ; \mathrm{c}, \mathrm{d})=\left([\mathrm{a}-\mathrm{c}]^{2}+[\mathrm{b}-\mathrm{d}]^{2}\right)^{1 / 2}$ and is expressed in $\mathrm{mm}$.

The displacement in the $\mathrm{x}$ and $\mathrm{y}$ axes was calculated in reference to the starting points $\mathrm{x}_{1}$ and $\mathrm{y}_{1}$ respectively. The $\mathrm{x}$ displacement of $d(\mathrm{x})=\mathrm{x}_{\mathrm{i}}-\mathrm{x}_{1}$ and $\mathrm{y}$ displacement

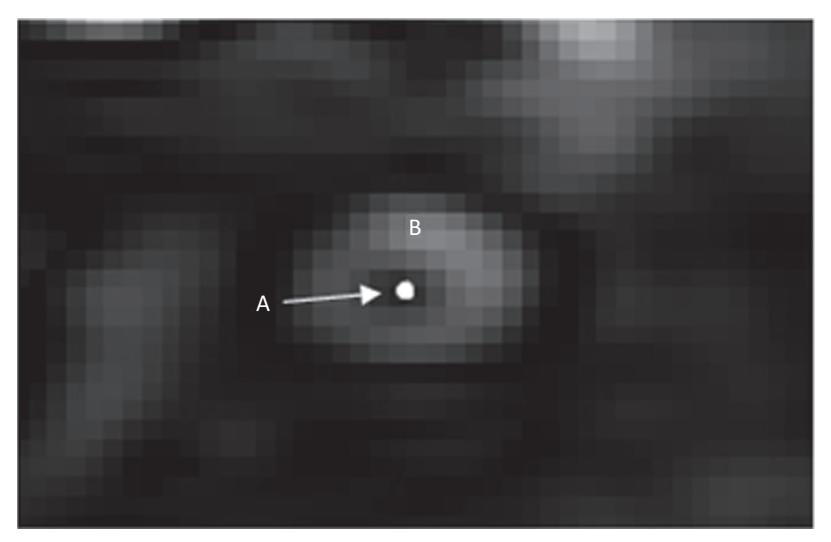

FIGURE 1: Echo-planar imaging snapshot of coronal oblique view of the righ optic nerve. A pixel-size target (white dot) was placed on the centre of the optic nerve and movement recorded over 3 minutes with 20 snapshots; (a), optic nerve; (b), peri-optic cerebrospinal fluid space. of $d(\mathrm{y})=\mathrm{y}_{\mathrm{i}}-\mathrm{y}_{1}$ for $\mathrm{i}=1-20$. Area of displacement was calculated as $(\max x-\min x) \times(\max y-\min y)$ and was expressed in $\mathrm{mm}^{2}$.

The ON slice approximately midway through the orbital segment of the nerve was used for diffusion analysis to match the segment chosen for movement analysis. It is the segment of the ON that is least susceptible to surrounding bone and air artifact, and is best visualised on diffusion imaging. A 2 × 2 pixel, square region of interest (ROI) was placed on the centre of the nerve from which diffusion parameters were obtained. The diffusion parameters (viz. ADC, MD, FA and AI) were obtained by imaging the ONs separately in the coronal oblique plane on DWI and DTI. ADC and AI were obtained from DWI whereas MD and FA were obtained from DTI. DWI acquisition was repeated in three orthogonal axes in order to obtain ADCs for each direction. The radial diffusion was the average of the two ADCs in the radial plane and compared with the axial diffusion when calculating the AI. Hence AI was calculated using the formula $\mathrm{AI}=2 \lambda_{1} /\left(\lambda_{2}+\lambda_{3}\right)$, where $\lambda_{1}=$ axial diffusion and $\lambda_{2}, \lambda_{3}=$ radial diffusion in the other two orthogonal axes (Table 2).

\section{Statistics}

Spearman's rank correlation was used to assess the association between movement and diffusion parameters owing to the small sample size, and the $t$-test to test for significance. Significance level was set at $p<0.05$. Statistical analysis was done in STATA, version 12.

\section{Results}

From the 29 healthy volunteers recruited, 26 right eyes and 26 left eyes were analysed for diffusion parameters owing to poor nerve visualisation in those excluded. Table 1 shows the demographic profile of the volunteers and the diffusion parameters recorded. Comparison is made with other reported diffusion sequences. Of the 29 volunteers, 20 had right optic nerve tracking recorded by the single-shot EPI method. The average total distance moved by the optic nerve during the 3 minutes' recording was $11.8 \mathrm{~mm}$ (range 5.7 23.7) despite requesting the patient to fixate on the orange sticker placed overhead on the MRI scanner (Table 2). Eye movement was perhaps a combination of voluntary and involuntary saccadic movement. Spearman's rank correlation showed low to moderate negative correlations between $\mathrm{ON}$ movement parameters and AI and FA of the ON (Table 2). Whilst the $t$-test did not reach significance levels, a trend towards this negative correlation was evident, especially in relation to ON displacement in the x-axis and AI (Table 2). No significant correlations were noted between any of the $\mathrm{ON}$ movement parameters and ADC in the axial plane and MD of the ON (Table 2).

Table 1 shows fairly similar diffusion parameters with and without CSF suppression, and the current values fall within the range of values that have been reported. 

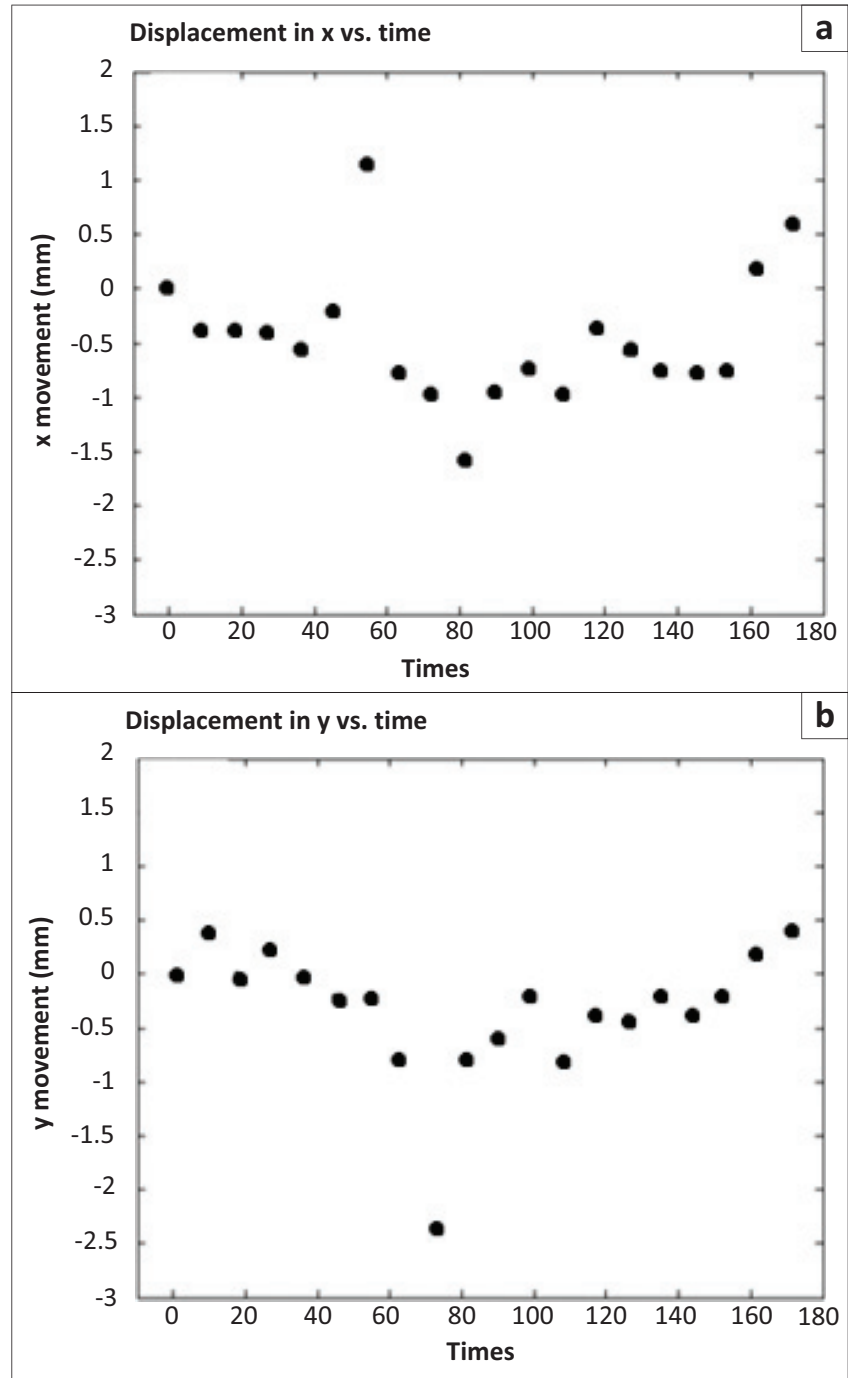

Displacement in y vs. Displacement in $\mathbf{x}$

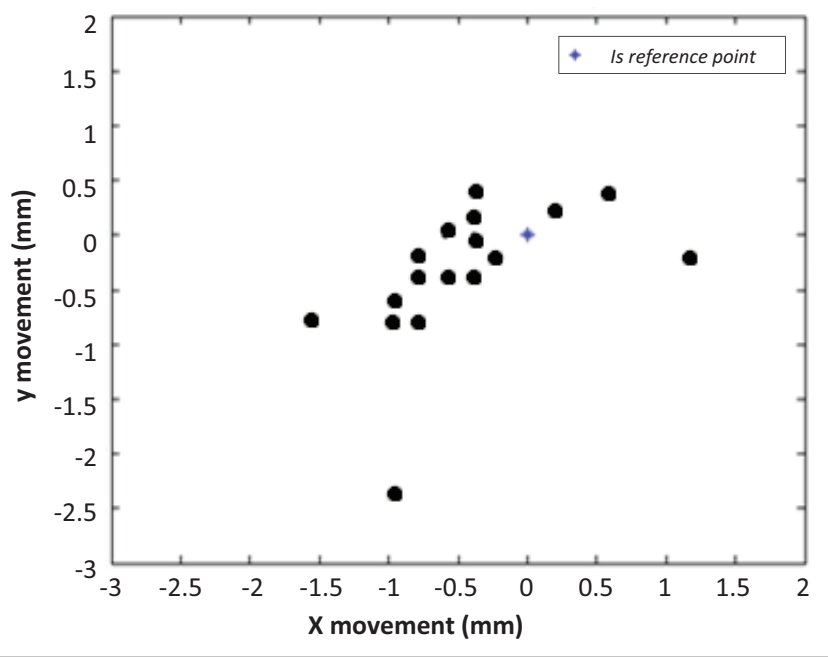

FIGURE 2: Sample displacement of the optic nerve in 20 snapshots in the $x$ and $y$ axes and the $x$-y plane from one subject; (a), x-axis; (b), y-axis; (c), x-y plane.

\section{Discussion}

ON diffusion as an investigational tool for $\mathrm{ON}$ microstructure in normal and pathological conditions is gaining in popularity. 2,5,6,11,12 Objective, reproducible and verifiable MR sequences for diffusion is paramount in maintaining this impetus. The influence of eye movement, however, remains a problem. Intuitively, one might tend to consider that $\mathrm{ON}$ movement affects the Brownian motion of water molecules within the nerve and consequently on $\mathrm{ON}$ diffusion imaging.

The range of movement between the immediate retrobulbar portion of the intra-orbital $\mathrm{ON}$ is far more than the precanalicular portion which is fixed. ${ }^{9}$ ON diffusion studies usually examine the mid-portion of the orbital ON which is relatively free from the susceptible artifacts of surrounding bone and air. ${ }^{1,413}$. The amount of movement by this portion of the nerve is therefore of relevance to the diffusion parameters measured in that region. Obsessive attempts to limit this movement invariably result in frustrated and less co-operative subjects. Wheeler-Kingshott et al. have shown that asking the subject to fixate on a point for 5 minutes or more does not significantly reduce eye movement. ${ }^{14}$

ON movement distorts diffusion imaging of the nerve. Six eyes had to be excluded from analysis owing to poor visualisation as a result of eye movement. Where analysis was possible, Table 1 shows that the results obtained were reliable. The $\mathrm{ADC}, \mathrm{MD}, \mathrm{FA}$ and $\mathrm{AI}$ of the ON obtained in the present study are comparable to those of Wheeler-Kingshott et al., Iwasawa et al. and Chabert et al. (Table 1).

Despite attempts to limit ON movement, both voluntary and involuntary saccadic movements are unavoidable during a DWI or DTI recording of between 3 and 5 minutes. Macroscopically, the $\mathrm{ON}$ moves appreciably during a 3 -minute recording. We recorded a mean total distance of $11.8 \mathrm{~mm}$ (range $5.7-23.7$ ) over a mean area of $5.2 \mathrm{~mm}^{2}$ (range 0.6 - 22.1). No significant correlation was noted between $\mathrm{ON}$ movement parameters and ADC in the axial plane and MD. However, low to moderate negative correlations were noted between $\mathrm{ON}$ movement parameters and FA and AI. The $t$-test showed a trend towards significance even though significance levels were not reached, which may be the result of the small sample size. Movement of the nerve, which physiologically occurs in the radial plane ( $x$ and $y$ displacement), did not significantly affect ADC in the axial plane and MD, but moderately increased ADC in the radial plane, resulting in less anisotropy of the nerve and subsequently a lower AI. Increased radial diffusion with ON movement also moderately lowered the FA even though significance levels were not reached. The moderate correlations of ON displacement in the $\mathrm{x}$ direction and ADC in the radial direction did not reach statistical significance ( $p=$ 0.17 ), but this too may be the result of the small sample size.

Surprisingly, not suppressing the peri-optic CSF had no significant effect on ON diffusion parameters from DWI and DTI in our series when compared with those where CSF suppression was done. The advantage of not suppressing the CSF signal is better identification of the nerve on $b=0 \mathrm{~s} / \mathrm{mm}^{2}$ images. An additional benefit is recording of peri-optic CSF diffusion parameters that can potentially be applied to disorders that involve the peri-optic CSF space, such as papilloedema.

A limitation of the present study was the inability to track movement of ONs during the actual performance of DWI 
TABLE 2: Optic nerve movement parameters and effect on diffusion parameters in 20 patients.

\begin{tabular}{|c|c|c|c|c|c|c|}
\hline \multirow[t]{4}{*}{ Optic nerve movement parameters } & \multicolumn{6}{|c|}{ Spearman's correlation between optic nerve movement and optic nerve diffusion parameters } \\
\hline & \multirow[t]{3}{*}{$M$ (range) / SD } & \multicolumn{2}{|c|}{ ADC } & \multirow{3}{*}{$\begin{array}{c}\text { MD } \\
\text { Optic nerve }\end{array}$} & \multirow{3}{*}{$\begin{array}{c}\text { FA } \\
\text { Optic nerve }\end{array}$} & \multirow{3}{*}{$\frac{\text { Al }}{\text { Optic nerve }}$} \\
\hline & & \multicolumn{2}{|c|}{ Optic nerve } & & & \\
\hline & & Radial & Axial & & & \\
\hline \multirow[t]{2}{*}{ Total distance moved $(\mathrm{mm})$} & 11.8 & - & - & - & - & - \\
\hline & $(5.7-23.7) / 3.45$ & 0.2 & 0.18 & 0.07 & -0.32 & -0.33 \\
\hline \multirow[t]{2}{*}{ Total area of displacement $\left(\mathrm{mm}^{2}\right)$} & 5.2 & - & - & - & - & - \\
\hline & $(0.6-22.1) / 3.75$ & 0.24 & 0.3 & 0.08 & -0.28 & -0.3 \\
\hline \multirow[t]{2}{*}{ Displacement in the $x$-axis ( $\mathrm{mm}$ ) } & 2.1 & - & - & - & - & - \\
\hline & $(0.8-5.1) / 0.97$ & 0.35 & 0.19 & 0.18 & -0.07 & $-0.45^{*}$ \\
\hline \multirow[t]{2}{*}{ Displacement in the $y$-axis $(\mathrm{mm})$} & 2.2 & - & - & - & - & - \\
\hline & $(0.7-7.5) / 1.61$ & 0.12 & 0.15 & -0.07 & -0.37 & -0.17 \\
\hline
\end{tabular}

$\mathrm{ADC}$, apparent diffusion coefficient measured using diffusion-weighted imaging; $\mathrm{MD}$, mean diffusivity measured using diffusion tensor imaging; $\mathrm{FA}$, fractional anisotropy; Al, anisotropic index calculated as $2 \lambda_{1} /\left(\lambda_{2}+\lambda_{3}\right)$, where $\lambda_{1}=$ axial diffusion and $\lambda_{2}, \lambda_{3}=$ radial diffusion; SD, standard deviation.

Spearman's correlation coefficient: Low or no correlation $0 \leq r<0.3$; moderate correlation $0.3 \leq r<0.7$; strong correlation $0.7 \leq r<1$

(- indicates negative correlation).

$*, p=0.07$.

and DTI of the ON. Nevertheless, we feel that the amount of ON movement was a good reflection of the mental and physical state of the subject as no time was wasted between imaging sequences. Both studies were done about 25 minutes into the entire MR imaging, when the subject was more settled. The concern of co-registration of diffusion volumes from the $\mathrm{ON}$ and surrounding CSF was addressed by the short scanning time with snapshots of the ON taken within 9 seconds. With the mean displacement of the ON during the entire 180 seconds being $2.1 \mathrm{~mm} \pm 0.97 \mathrm{~mm}$ in the $x$-axis and $2.2 \mathrm{~mm} \pm 1.61 \mathrm{~mm}$ in the $\mathrm{y}$-axis, one could predict that with each image acquisition over a 9-second interval, the $\mathrm{ON}$ would have spent most of the time close to or within the reference point (Table 2). The standard deviations given are of the order of 1 pixel in size. The ON is normally $4 \mathrm{~mm}-6 \mathrm{~mm}$ in diameter, therefore manual placement of the $2 \times 2$ pixel ROI within the nerve also avoided co-registration of diffusion volumes between the $\mathrm{ON}$ and surrounding CSF.

Our study provides reasonable evidence that macroscopic ON movement has an effect on radial diffusion obtained from the mid-portion of the orbital segment of the ON. The result is lowering of the $\mathrm{AI}$ and FA. Consequently, future studies that evaluate anisotropic diffusion of the ON need to take into account the effect of $\mathrm{ON}$ movement and motion correction methods or software devised to reduce the errors. MD, as expected, is unaffected by this movement as it is a scalar average of the diffusion tensor. Furthermore, recording of ON movement as obtained with rapid sequential singleshot EPI is valid and a reasonable reflection of the amount of eye movement during standard MR sequences.

\section{Acknowledgements Competing interests}

The authors declare that they have no financial or personal relationship(s) that may have inappropriately influenced them in writing this article.

\section{Authors' contributions}

A.M. (University of KwaZulu-Natal), W.R. (University of the Free State), A.B. (University of KwaZulu-Natal) and
Y.B. (University of KwaZulu-Natal) were responsible for the study conception and design. A.M., A.M. (Edendale Hospital) and Y.B. did acquisition of data. A.M., Y.B., W.R., A.B., M.D. (Grey's Hospital) and C.C. (Medical Research Council) did the analysis and interpretation of the data. A.M. and W.R. drafted the manuscript, and W.R., A.B. and M.D. did the critical revision.

\section{References}

1. Xu J, Sun SW, Naismith RT, Snyder AZ, Cross AH, Song SK. Assessing optic nerve pathology with diffusion MRI: From mouse to human. NMR Biomed. 2008;21(9):928-940. http://dx.doi.org/10.1002/nbm.1307

2. Trip SA, Wheeler-Kingshott $C$, Jones SJ, et al. Optic nerve diffusion tensor imaging in optic neuritis. Neuroimage. 2006;30(2):498-505. http://dx.doi. org/10.1016/j.neuroimage.2005.09.024

3. Zhang $Y$, Wan SH, Wu GJ, Zhang XL. Magnetic resonance diffusion tensor imaging and diffusion tensor tractography of human visual pathway. Int J Ophthalmol. 2012;5(4):452-458.

4. Fatima Z, Motosugi U, Muhi A, Hori M, Ishigame K, Araki T. Diffusion-weighted imaging in optic neuritis. Can Assoc Radiol J. 2013;64(1):51-55. http://dx.doi. org/10.1016/j.carj.2011.08.006

5. Cauquil C, Souillard-Scemama R, Labetoulle M, Adams D, Ducreux D, Denier C. Diffusion MRI and tensor tractography in ischemic optic neuropathy. Acta Neuro Belg. 2012;112(2):209-211. http://dx.doi.org/10.1007/s13760-012-0013-5

6. Chen Z, Lin F, Wang J, et al. Diffusion tensor MRI reveals visual pathway damage that correlates with clinical severity in glaucoma. Clin Experiment Ophthalmol. 2013;41(1):43-49. http://dx.doi.org/10.1111/j.1442-9071.2012.02832.x

7. Moodley A, Rae W, Bhigjee A, Loubser N, Michowicz A. New insights into the pathogenesis of cryptocococcal induced visual loss using diffusion-weighted imaging of the optic nerve. Neuro-Ophthalmology. 2012;36(5):186-192. http:// dx.doi.org/10.3109/01658107.2012.715716

8. Iwasawa $T$, Matoba $H$, Ogi $A$, et al. Diffusion-weighted imaging of the human optic nerve: A new approach to evaluate optic neuritis in multiple sclerosis. Magn Reson Med. 1997;38(3):484-491. http://dx.doi.org/10.1002/mrm.1910380317

9. Wheeler-Kingshott CA, Parker GJ, Symms MR, et al. ADC mapping of the human optic nerve: Increased resolution, coverage, and reliability with CSF-suppressed ZOOM-EPI. Magn Reson Med. 2002;47(1):24-31. http://dx.doi.org/10.1002/ mrm.10016

10. Chabert S, Molko N, Cointepas $Y$, Le Roux P, Le Bihan D. Diffusion tensor imaging of the human optic nerve using a non-CPMG fast spin echo sequence. J Magn Reson Imaging. 2005;22(2):307-310. http://dx.doi.org/10.1002/jmri.20383

11. Hickman SJ, Wheeler-Kingshott CA, Jones SJ, et al. Optic nerve diffusion measurement from diffusion-weighted imaging in optic neuritis. AJNR Am J Neuroradiol. 2005;26(4):951-956.

12. Kolbe $S$, Chapman $C$, Nguyen T, et al. Optic nerve diffusion changes and atrophy jointly predict visual dysfunction after optic neuritis. Neuroimage. 2009;45(3):679686. http://dx.doi.org/10.1016/j.neuroimage.2008.12.047

13. Naismith RT, Xu J, Tutlam NT, Trinkaus K, Cross AH, Song SK. Radial diffusivity in remote optic neuritis discriminates visual outcomes. Neurology 2010;74(21):17021710. http://dx.doi.org/10.1212/WNL.0b013e3181e0434d

14. Wheeler-Kingshott CA, Trip SA, Symms MR, Parker GJ, Barker GJ, Miller DH. In vivo diffusion tensor imaging of the human optic nerve: pilot study in normal controls. Magn Reson Med. 2006;56(2):446-451. http://dx.doi.org/10.1002/mrm.20964 